\title{
Estudo retrospectivo da prevalência de raiva bovina no Estado de Rondônia e sua distribuição entre os anos de 2009 e 2018
}

\author{
Background study on the prevalence of bovine rabies in the State of Rondonia and its distribution \\ between 2009 and 2018 \\ Estudio retrospectivo de la prevalencia de rabia bovina en el Estado de Rondônia y su distribución
}

entre 2009 y 2018

Recebido: 08/07/2021 | Revisado: 18/07/2021 | Aceito: 20/07/2021 | Publicado: 28/07/2021

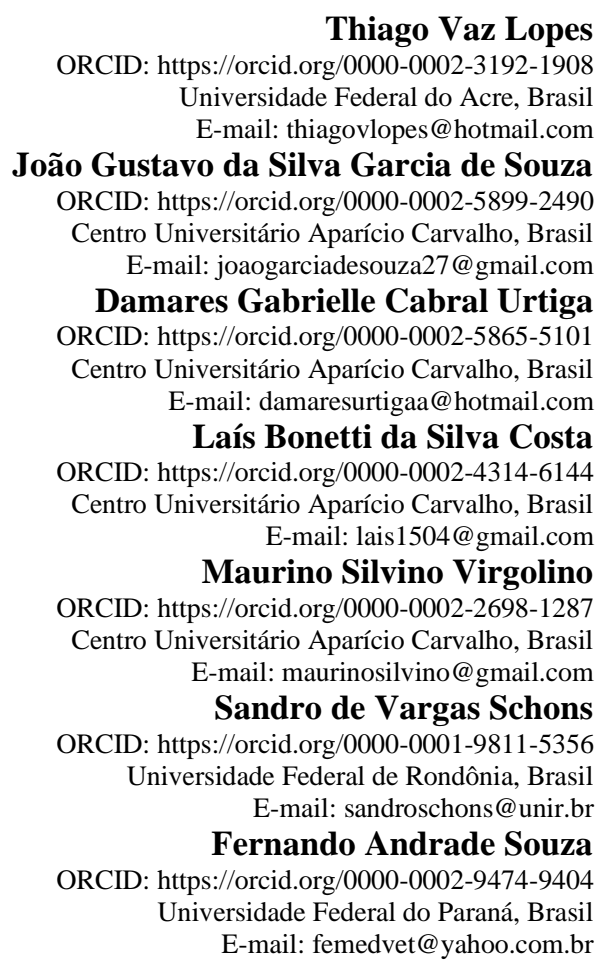

\section{Resumo}

A Raiva nos herbívoros, disseminada principalmente pelo morcego hematófago Desmodus rotundus, que transmite através da mordedura o vírus Rhabdoviridae, é uma das doenças neurológicas mais acometidas no rebanho bovino brasileiro. Contudo, esse artigo tem como objetivo realizar um estudo retrospectivo da raiva em herbívoros no estado de Rondônia, Brasil, avaliando os dados cedidos pela agência de defesa sanitária do estado no período compreendido de 2009 a 2018, neste espaço de tempo foi submetido a testes de imunofluorescência direta (IFD) 1.005 animais, dentre eles 47 resultantes positivos, equivalente a cerca $4,7 \%$ do total dos bovinos verificados. Ao decorrer do tempo, percebesse que o número de animais testados foi-se diminuindo juntamente com a quantidade de animais positivos para patologia, onde no ano de 2009 foi constatado 15 casos com a realização de 106 testes e já nos próximos 3 anos com a realização de 321 teste resultaram apenas 10 confirmações. Isso demonstra a eficiência da aplicação do programa nacional de controle da raiva dos herbívoros pelo órgão competente.

Palavra-chave: Raiva bovina; Rondônia; Estudo retrospectivo; Doença neurológica; Desmodus rotundos; Imunofluorescência direta.

\begin{abstract}
Herbivore rabies, mainly disseminated by the hematophagous bat Desmodus rotundus, which transmits through the bite the virus Rhabdoviridae, is one of the most affected neurological diseases in the Brazilian cattle herd. However, this article aims to carry out a retrospective study of rabies in herbivores in the state of Rondônia, Brazil, evaluating the data provided by the health defense agency os state from 2009 to 2018, in this period of time. direct immunofluorescence tests 1,005 animals, 47 of them positive results, equivalent to about $4.7 \%$ of the total cattle verified. Over time, it became clear that the number of animals tested was decreasing along with the number of positive animals for pathology, where
\end{abstract}


in 2009 there were 15 cases with 106 tests and already in the next 3 years with out of 321 tests resulted in only 10 confirmations. This demonstrates the effectiveness of application of the national herbivore rabies control program.

Keywords: Bovine rabies; Rondônia; Retrospective study; Neurological disease; Desmodus rotundus; Direct immunofluorescence.

\section{Resumen}

La rabia en herbívoros, transmitida principalmente por el murciélago vampiro Desmodus rotundus, que transmite el virus Rhabdoviridae a través de las mordeduras, es una de las enfermedades neurológicas más afectadas en el ganado brasileño. Sin embargo, este artículo tiene como objetivo realizar un estudio retrospectivo de la rabia en herbívoros en el estado de Rondônia, Brasil, evaluando los datos proporcionados por la agencia estatal de defensa de la salud en el período de 2009 a 2018. Inmunofluorescencia directa (DIF) 1.005 animales, incluidos 47 resultados positivos, lo que equivale aproximadamente al $4,7 \%$ del total de bovinos verificados. Con el tiempo, me di cuenta de que la cantidad de animales probados iba disminuyendo junto con la cantidad de animales positivos para la patología, donde en 2009 se encontraron 15 casos con la realización de 106 pruebas y ya en los próximos 3 años con la realización de 321 pruebas. resultó sólo 10 confirmaciones. Esto demuestra la eficiencia de la aplicación del programa nacional de control de la rabia en herbívoros por parte de la agencia competente.

Palabra clave: Rabia bovina; Rondônia; Estudio retrospectivo; Enfermedad neurológica; Desmodus rotundo; Inmunofluorescencia directa.

\section{Introdução}

O período histórico da raiva tem início no ano de 350 a.C., neste período essa doença já era considerada como patologia contagiosa e, a partir das mordidas de cães, era temida pelas pessoas e conhecida como uma doença mortal (Alves et al. 2020). Considerada uma das zoonoses mais antigas já descritas, que se tem conhecimento atualmente (Babbonia \& Modolo, 2011; Santos, 2018).

Estima-se que na América Latina a raiva seja responsável por grandes prejuízos econômicos, com a morte de 40.000 cabeças de gado/ano, com um déficit de aproximadamente 15 milhões de dólares (Teixeira, et al. 2015), sendo também endêmica na região norte do país (Ribeiro, et al. 2018; Ribeiro, 2017). Sendo considerada uma das doenças neurológicas mais acometidas em bovinos no Brasil, a raiva corre pelo vírus da família Rhabdoviridae, gênero Lyssavirus, da espécie rabies vírus (RABV). A transmissão do vírus acontece com mais frequência pelo morcego hematófago Desmodus rotundus, facilmente encontrado em cavernas, ocos de árvores, minas e casas abandonadas (Laredo, 2015; Silva et al. 2019; Barroso et al. 2018).

Após o período de incubação, os sinais clínicos começam a se apresentar, não sendo patognomônicos. A observação clínica indicará apenas a suspeita da enfermidade acometendo o sistema nervoso central dos animais. Não existe tratamento para a doença e, uma vez iniciados os sinais clínicos, é invariavelmente fatal (Brasil, 2008). Contudo, animais que apresentam sinais clínicos suspeitos devem obrigatoriamente ser notificados pelo médico veterinário aos órgãos de defesa animal, e submetidos a necropsia para confirmação de diagnóstico (OIE, 2019).

Após a colheita do sistema nervoso central do animal com suspeita de infecção, o material deverá ser processado por meio da técnica de imunofluorescência direta (IFD) e prova biológica (PB), que se dá pela inoculação em camundongos ou células ou outra técnica que, porventura, seja recomendada pela Organização Mundial da Saúde (OMS) em laboratório oficial ou privado, credenciados pelo ministério da agricultura, pecuária e abastecimento - MAPA (MAPA, 2005).

O presente trabalho tem como objetivo central determinar a distribuição no espaço-tempo, em um período de dez anos dos resultados positivos em testes realizados para confirmação da raiva em grandes animais no estado de Rondônia, com o intuito de determinar a prevalência desse período.

\section{Material e Métodos}

O estado de Rondônia possui uma área compreendida por $237.765,233 \mathrm{~km}^{2}$, fazendo fronteira ao norte com o estado do amazonas, oeste com o acre, leste com o mato grosso e na região sudoeste faz fronteira internacional com a Bolívia. Os dados 
de raiva bovina e bubalina diagnostica dentro desse território estão armazenados em um banco de dados no órgão de defesa animal do estado de Rondônia, sendo a partir deste o processamento e análise para a presente pesquisa.

Os dados foram coletados e tabulados em planilha do Microsoft Excel@ 2007, afim de realizar um levantamento de dados, de forma quantitativa, contendo o número dos animais testados no período dos anos de 2009 a 2018, sendo descrito os municípios em que foram registrados os casos, as espécies acometidas, compreendidas por equinos, bovinos, bubalinos e ovinos e caprinos, como também se havia registros do envolvimento de morcegos hematófagos ou não hematófagos na descrição dos casos, também foram colheitados os dados referentes aos resultados dos testes do exame parcial e do confirmatório.

Foram considerados os animais para estudo os que apresentaram como positivos, através das informações de formulários de investigação (form in) estabelecido pela Organização Mundial de Saúde Animal-OMS, no período de 2009 a 2018, sendo foram considerados como ocorrência de um foco de raiva a partir de testes laboratoriais confirmatório.

$\mathrm{Na}$ avaliação dos cômputos, o critério utilizado foi a observação do resultado dos testes imunofluorescência direto-IFD (parcial) e a prova biológica -PB (final), os animais positivos para o teste parcial, mas negativos para o final ou os animais que foram reagentes positivos ao teste parcial, mas porventura não realizam a prova biológica, não foram contabilizados como caso confirmado, logo, todos os reagentes positivos para algum dos testes foram estabelecidos como caso suspeito.

\section{Resultados}

Os resultados totalizaram exatamente 1.005 animais testados, dentre eles, 47 (4,68\%) animais foram constatados reagentes positivos para algum dos dois testes, o de imunofluorescência direta-IFD utiliza imunoglobulinas antirrábicas marcadas com isotiocianato de fluoresceína, sendo assim, um teste rápido ou no teste de Prova Biológica - PB, que é o teste confirmatório (Brasil, 2008).

Dentre os 47 animais reagentes suspeitos, $41 / 47$ (87,23\%) foram diagnosticados pelo IFD sendo confirmatórios também no teste PB, $6 / 47$ (12,77\%) foram positivas apenas no teste IFD não sendo realizado o teste PB, devido ao elevado grau de autólise, sendo a distribuição desses em 21 cidades dentre as 52 do estado de Rondônia

Em relação aos anos estudados frente as manifestações desta patologia, pode-se observar m 2009 o maior número de casos suspeitos e confirmados (Gráfico 1), com 15/47 casos suspeitos e 13/41 casos confirmados, apresentando uma prevalência de 12,3\% do total de testes, seguidos pelos anos de 2010 com 8,1\% de confirmações e 2013 com 5,9\% de casos confirmados. Entretanto, nos anos de 2014 a 2018, os índices não sofreram alterações consideráveis, em média 2 casos confirmados por ano, sendo que nos anos de 2015 e 2016 não obtiveram resultados confirmatórios através da prova biológica, porém com 3 casos no total positivos para o teste de imunofluorescência direta. 
Gráfico 1 - Resultados parciais e confirmatórios de raiva em grandes animais durante os anos de 2009 a 2018 , no estado de Rondônia.

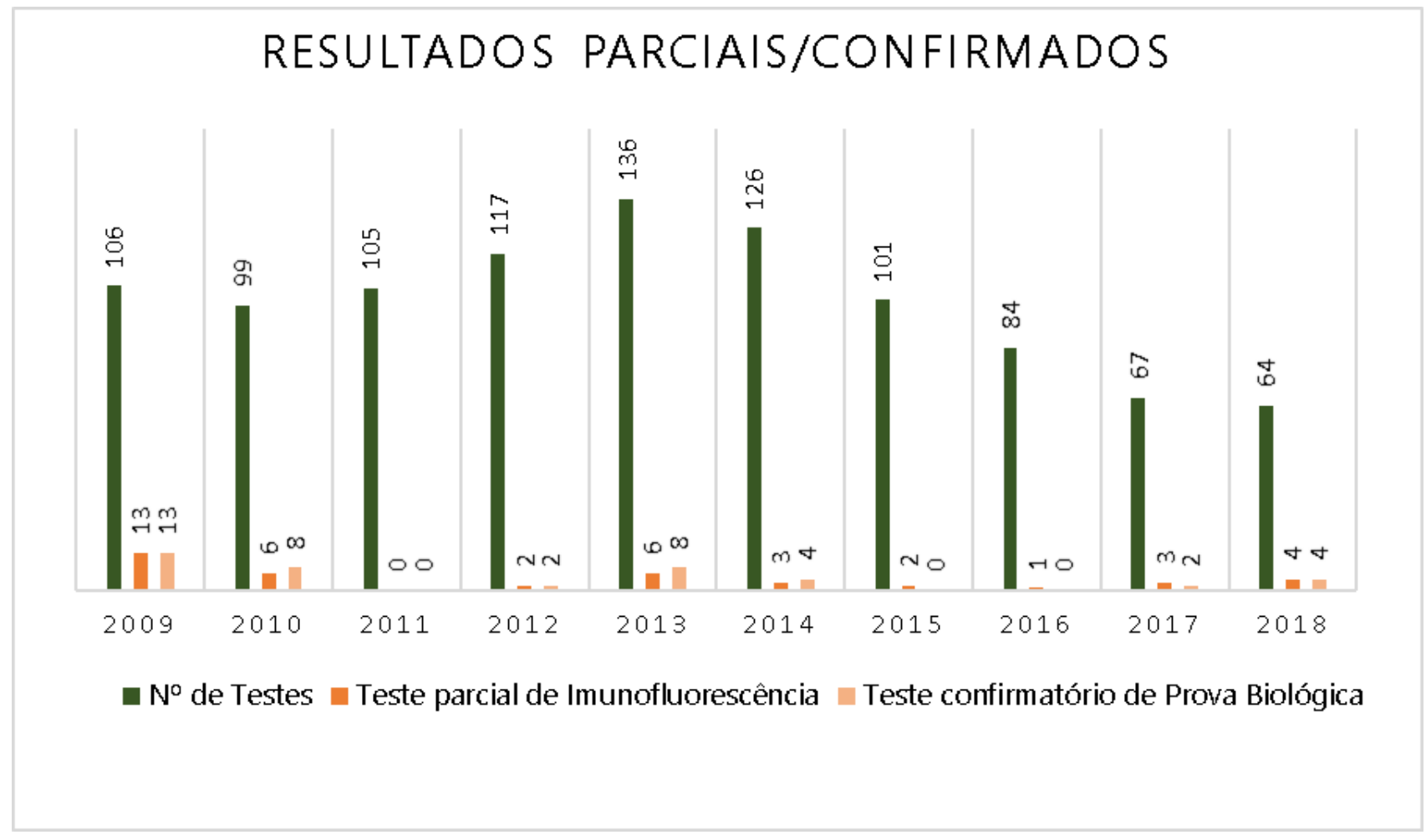

Fonte: Órgão de defesa sanitária do estado de Rondônia (2019).

Dentre os 52 municípios do estado de Rondônia, 21 deles apresentaram a manifestação de casos de raiva em animais de produção, durante o período estudado, sendo todos os diagnósticos em bovinos, sendo os municípios com maior prevalência de raiva bovina as cidades de: Vilhena 5/47 (10,63\%); Ariquemes 5/47 (10,63\%); São Miguel do Guaporé 5/47 (10,63\%); Porto Velho 4/47 (8,51\%); Cacoal 4/47 (8,51\%); Espigão D’oeste 3/47 (6,38\%); Nova Brasilândia 3/47 (6,38\%); Alta floresta D’oeste 3/47 (6,38\%); (Tabela 1) totalizado em 2018 apenas nessas cidades com os maiores números de acometimentos, uma população bovina de 3.413.735, levando em consideração que em todo o estado havia nesse ano 14.367 .161 , cerca de $23,8 \%$ estão localizados nos municípios descritos, outro fator que pode ter influenciado no número de casos, são os altos números de abrigos naturais para o principal vetor do vírus, o morcego hematófago desmodus rotundos. 
Tabela 1 - Relação dos municípios do estado de Rondônia que apresentaram resultados de imunofluorescência direta - IFD ou/e prova biológica-PB para raiva em animais de produção, durante os anos de 2009 a 2018.

\begin{tabular}{|c|c|c|c|c|}
\hline ANO & MUNICÍPIO & ESPÉCIE & IFD & PB \\
\hline 2014 & Alto Alegre dos Parecis & Bovino & Negativo & Positivo \\
\hline 2009 & Alta Floresta do Oeste & Bovino & Positivo & - \\
\hline 2009 & Alta Floresta do Oeste & Bovino & Positivo & Positivo \\
\hline 2010 & Alta Floresta d' Oeste & Bovino & Negativo & Positivo \\
\hline 2010 & Ariquemes & Bovino & Positivo & Positivo \\
\hline 2010 & Ariquemes & Bovino & Negativo & Positivo \\
\hline 2010 & Ariquemes & Bovino & Positivo & Positivo \\
\hline 2010 & Ariquemes & Bovino & Positivo & Positivo \\
\hline 2017 & Ariquemes & Bovino & Positivo & Positivo \\
\hline 2009 & Buritis & Bovino & Positivo & - \\
\hline 2009 & Buritis & Bovino & Positivo & Positivo \\
\hline 2010 & Cabixi & Bovino & Positivo & Positivo \\
\hline 2014 & Cacoal & Bovino & Positivo & Positivo \\
\hline 2014 & Cacoal & Bovino & Positivo & Positivo \\
\hline 2015 & Cacoal & Bovino & Positivo & - \\
\hline 2015 & Cacoal & Bovino & Positivo & - \\
\hline 2009 & Chupinguaia & Bovino & Negativo & Positivo \\
\hline 2009 & Corumbiara & Bovino & Positivo & Positivo \\
\hline 2010 & Espigão do Oeste & Bovino & Positivo & Positivo \\
\hline 2013 & Jaru & Bovino & Positivo & Positivo \\
\hline 2013 & Jaru & Bovino & Positivo & Positivo \\
\hline 2013 & Jaru & Bovino & Positivo & Positivo \\
\hline 2013 & Espigão do Oeste & Bovino & Negativo & Positivo \\
\hline 2013 & Espigão do Oeste & Bovino & Positivo & Positivo \\
\hline 2013 & Mirante da Serra & Bovino & Positivo & Positivo \\
\hline 2009 & Nova Brasilândia & Bovino & Positivo & Positivo \\
\hline 2009 & Nova Brasilândia & Bovino & Positivo & Positivo \\
\hline 2009 & Nova Brasilândia & Bovino & Positivo & Positivo \\
\hline 2009 & Ouro Preto do Oeste & Bovino & Positivo & Positivo \\
\hline 2009 & Porto Velho & Bovino & Positivo & Positivo \\
\hline 2009 & Porto Velho & Bovino & Negativo & Positivo \\
\hline 2013 & Porto Velho & Bovino & Negativo & Positivo \\
\hline 2017 & Porto Velho & Bovino & Positivo & Positivo \\
\hline 2017 & Rolim de Moura & Bovino & Positivo & -- \\
\hline 2012 & Santa Luzia d'Oeste & Bovino & Positivo & Positivo \\
\hline 2013 & São Francisco do Guaporé & Bovino & Positivo & Positivo \\
\hline 2009 & São Miguel do Guaporé & Bovino & Positivo & Positivo \\
\hline 2010 & São Miguel do Guaporé & Bovino & Positivo & Positivo \\
\hline 2012 & São Miguel do Guaporé & Bovino & Positivo & Positivo \\
\hline 2013 & São Miguel do Guaporé & Bovino & Positivo & Positivo \\
\hline 2016 & São Miguel do Guaporé & Bovino & Positivo & - \\
\hline 2009 & Vale do Anari & Bovino & Positivo & Positivo \\
\hline
\end{tabular}




\begin{tabular}{lllll}
2009 & Vilhena & Bovino & Positivo & Positivo \\
2018 & Vilhena & Bovino & Positivo & Positivo \\
2018 & Vilhena & Bovino & Positivo & Positivo \\
2018 & Vilhena & Bovino & Positivo & Positivo \\
2018 & Vilhena & Bovino & Positivo & Positivo \\
\hline
\end{tabular}

Fonte: Com base nos dados analisados nessa pesquisa.

\section{Discussão}

Dentre os animais de produção analisados com suspeita de raiva em um período de 10 anos no estado de Rondônia, foram observados uma positividade de 4,68\%, estando esses valores de encontro aos estabelecidos por Dognani et al. (2016), em que estabeleceu uma prevalência de $28,1 \%$ no estado do Pará, a diferença entre o tamanho da população bovina dos dois estados não é tão discrepante quantos os valores das prevalências, podendo ser justificado devido a esse estudo estar tratando de 10 anos contra 36 para o trabalho deles, sendo parte desse tempo um período bem expressivo em que não se havia no brasil um programa nacional de controle da raiva em herbívoros (Brasil, 2002).

O avanço da pecuária segundo Carvalho et al. (2009) com associado a degradação de áreas naturais, tem levado a desequilíbrios ambientais e proporcionando o encontro do agente e hospedeiro nesse ambiente, fechando o então denominado de ciclo rural da raiva, sendo essa a realidade do estado de Rondônia, ficando evidente a manifestação da raiva nas regiões do estado com maior avanço da pecuária nos últimos anos

No estado do Tocantins (Lopes et al. 2010) nesse mesmo período estudo foram colheitadas amostras de 341 animais com suspeita de raiva, número bem inferiores aos 1005 dessa pesquisa em que apenas 47 foram positivos, porém lá positivaram 36,65, valores bem superiores quando comparado aos 4,68\% encontrados nessa pesquisa (Tocatins, 2011; Oliveira et al. 2013).

A transmissão da raiva no ciclo rural tem como fonte e agente transmissor o morcego Desmodus rotundus que é o principal transmissor entre os hematófagos. Alguns trabalhos também relatam a infecção via inalação, sendo comum pelo contato das cavernas com presença de secreções de morcegos portadores do vírus (Megid et al. 2016)

Para o diagnostico então tanto dos morcegos, herbívoros ou até mesmo em humanos, não se tem um exame especifico laboratorial conclusivo para ser feito no ante mortem, porém após a morte existem protocolos para a avaliação de tecidos do sistema nervoso central, que são examinados afim de confirmar o caso de raiva através de uma prova biológica, que consiste na inoculação de secreção em camundongos com acompanhamento da manifestação da raiva também nesses animais, confirmando assim caso venha a manifestar, uma barreira para esse teste é o processo de autólise que dar-se inicio logo após a morte, sendo descrito nos resultados dessa pesquisa um número de 6/47 (12,76\%) das amostras analisadas em que essa alteração impossibilitou a análise conclusiva (Brasil, 2010).

\section{Conclusão}

Concluisse que ocorreram manifestação de casos de raiva no estado de Rondônia no período estudado, perfazendo uma prevalência de 4,68\% de casos dentre os casos suspeitos com abertura de Form-in.

\section{Referências}

Alves, A. L. et al. (2020). Raiva bovina: revisão. PUBVET, 14(7): 1-3.

Babboni, S. D. \& Modolo, J. R. Raiva: Origem, Importância e Aspectos Históricos. UNOPAR Cientifica. Ciências Biológicas e da Saúde 2011;13(Esp):349-56.

Barroso, R. M. V. et al. (2018). Ocorrência de casos positivos de raiva em herbívoros na região rural de Colatina-Brasil nos anos de 2013 a 2015. REDVET, $19(1)$. 
Brasil. (2002). Instrução Normativa $n^{\circ} 5$, de $1^{\circ}$ de março de 2002. Aprova as normas técnicas para o controle de raiva dos herbívoros domésticos, em conformidade com o anexo a esta instrução normativa. Diário Oficial da República Federativa do Brasil, Brasília, DF, n. 42, Seção 1, 2002.

Brasil. MINISTÉRIO DA SAÚDE. (2010). Secretaria de Vigilância em Saúde. Departamento de Vigilância Epidemiológica. Guia de Vigilância Epidemiológica. Brasília: Ministério da Saúde.

Brasil. (2018). Pecuária. Instituto Brasileiro de Geografia e Estatística, 2018. <https://cidades.ibge.gov.br/brasil/ro/pesquisa/18/16459>.

Carvalho, J. A. et al. (2009). Doenças Emergentes: uma Análise Sobre a Relação do Homem com o seu Ambiente. Revista Práxis, 1: $19-23$.

Laredo, G. (2015) Morcego que dá raiva. Revista Globo rural. 2015. <http://revistagloborural.globo.com/GloboRural/0,6993,EEC550250-2584,00.html>.

Lopes, A. G. (2010). Incidência da Raiva dos Herbívoros em Bovinos no Estado do Tocantins. In: VI SEMINÁRIO DE INICIAÇÃO CIENTÍFICA DA UFT. Palmas-TO. Anais. Palmas: UFT. 1 CDROM.

MAPA. (2009). Programa Nacional de Controle da Raiva dos Herbívoros. Manual de Legislação - Programas Nacionais de saúde animal do Brasil, 2009. p. 144. <file://C:/Users/mauri/Downloads/Manual\%20de\%20Legisla\%C3\%A7\%C3\%A3o\%20-\%20Sa\%C3\%BAde\%20Animal\%20-\%20low\%20(1).pdf>.

Megid, J. et al. (2016). Doenças infecciosas em animais de produção e de companhia. Roca. 799-821 p.

Monike S. O. et al. (2013). Frequência Da Raiva Em Herbívoros E Humanos No Estado Do Tocantins De 1999 A 2010 : Relatório Técnico. Acta Veterinaria Brasilica, 7(3): 180-183.

OIE. Organização Mundial de Saúde Animal. <http://www.oie.int.>.

Rondônia (2017). Rebanho bovino ultrapassa 14 milhões de cabeças em Rondônia. Agência de Defesa Sanitária Agrosilvopastoril, 2017. <http://www.rondonia.ro.gov.br/rebanho-bovino-ultrapassa-14-milhoes-de-cabecas-em-rondonia/>.

Ribeiro, T. M. P. et al. (2018). Casos notificados de raiva em bovinos na região norte no período de 2005-2017. Jornal Interdisciplinar de Biociências, 3(1).

Ribeiro, T. M. P. (2017). Casos e Focos de Raiva e Brucelose em Bovinos (Bos taurus) registrados em Roraima no Período 2008-2016. Bol. Mus. Int. de Roraima., 11(2): 49-54.

Santos, G. B. (2018). Raiva Bovina: Revisão De Literatura. 24f. Dissertação para conclusão de curso. Universidade Federal do Rio Grande do Sul - Faculdade de Veterinária, Porto Alegre.

Silva, E. M. C. et al. (2019). Estudo retrospectivo da raivia em herbívoros e animais silvestres no estado do Maranhão. ARS VETERINARIA, 35(2): 056-062.

Teixeira, L. H. M. et al. (2015). Distribuição Espaço-Temporal Dos Diagnósticos Laboratoriais Da Raiva Animal. Cienc. anim. bras. 16(1): 144-157.

Tocantins. (2011). Agência de Defesa Agropecuária do Tocantins. Portaria n.94, de 13 de abril de 2011. Torna recomendável a vacinação contra raiva nos municípios de maior risco no Tocantins a partir de maio de 2011. Diário Oficial do Estado do Tocantins, Palmas, TO, n. 3.363 de 15/04/2011, p. 63. 